\title{
LACTATE AND GAS EXCHANGE RESPONSES TO INCREMENTAL AND STEADY STATE RUNNING
}

\author{
M. HAVERTY, W. L. KENNEY and J. L. HODGSON \\ Laboratory for Human Performance Research, The Pennsylvania State University
}

\section{ABSTRACT}

This study compared the oxygen uptake $\left(\mathrm{VO}_{2}\right)$ and running velocity at which the lactate threshold (LT), the ventilatory threshold (VT), and the maximal lactate steady state (MSSLA), and the maximal $\mathrm{VO}_{2}$ steady state $\left(\mathrm{MSSVO}_{2}\right)$ occurred in 11 trained male runners (mean age $=22.4$ years, range $18-28$ years). Each underwent an incremental treadmill test to exhaustion. The LT was defined by a systematic, continuous increase in arterialised venous blood lactate; the VT was determined by an abrupt rise in $\mathrm{VE} . \mathrm{VO}_{2}{ }^{-1}$ without an increase in $\mathrm{VE}^{\mathrm{V}} \mathrm{VCO}_{2}{ }^{-1}$. Each subject also completed a series of steady state treadmill runs of 20 minutes duration. The MSSLA was determined as the highest velocity and $\mathrm{VO}_{2}$ at which lactate concentration increased by less than 0.2 mmol. I $^{-1}$ from minute 10 to minute 20 . The $\mathrm{MSSVO}_{2}$ was determined as the highest velocity or $\mathrm{VO}_{2}$ at which a steady state in $\mathrm{VO}_{2}$ was not delayed for more than 3 minutes (with a steady state defined as $\mathrm{VO}_{2}$ within 0.2 I.min-1 of the average $\mathrm{VO}_{2}$ over the last 10 minutes of each test). Each subject also completed a $5 \mathrm{~km}$ time trial run to assess performance. No significant differences were found among the four variables expressed either as $\mathrm{VO}_{2}$ or velocity. Significant correlations were found between MSSLA and $\mathrm{MSSVO}_{2}(r=0.74)$ expressed as $\mathrm{VO}_{2}$, and between $\mathrm{MSSLA}$ and $\mathrm{MSSVO}_{2}(r=0.90), \mathrm{MSSVO}_{2}$ and $V T(r=0.70)$ and MSSLA and $V T(r=0.67)$ expressed as velocity. A stepwise regression analysis found MSSLA (expressed as velocity) to be the best predictor of $5 \mathrm{~km}$ performance $(r=0.87)$. It was concluded that (a) $\mathrm{MSSLA}$ and $\mathrm{MSSVO}_{2}$ are closely related, and (b) MSSLA is a good predictor of performance and may be an important, objective measure of cardiorespiratory endurance capacity.

Key Words: Lactate threshold, Ventilatory threshold, Anaerobic threshold, Maximal lactate steady state, Maximal $\mathrm{VO}_{2}$ steady state

\section{INTRODUCTION}

Cardiorespiratory endurance has traditionally been quantified by measuring maximum oxygen consumption $\left(\mathrm{VO}_{2} \mathrm{max}\right)$. The phenomenon described as the anaerobic threshold has been suggested to be a better measure of cardiorespiratory endurance capacity by Wasserman et al (1973), yet there has been a great deal of debate about this concept and methods of measurement. The anaerobic threshold has been estimated by many investigators using both blood lactate measurements (lactate threshold, LT) and gas exchange measurements (ventilatory threshold, VT), and the two tend to be intercorrelated (Caizzo et al, 1982; Davis et al, 1976; Ivy et al, 1980; Kumagai et al, 1982; Reinhard et al, 1979).

The LT has most often been measured as a systematic increase in blood lactate concentration determined subjectively. Inconsistent methods of blood sampling using venous (e.g. Davis et al, 1976, 1979; Ivy et al, 1981)), arterial (e.g. Green et al, 1983), and arterialised venous (e.g. Forster et al, 1972, McLellan, 1985) samples have made comparison of studies difficult.

The VT has also been measured by several different criteria, including a non-linear increase in $\mathrm{VE}$ and $\mathrm{VCO}_{2}$, an abrupt rise in $\mathrm{FEO}_{2} \%$, or an abrupt increase in $\mathrm{VE} \mathrm{VO}_{2}{ }^{-1}$ without an increase in $\mathrm{VE} . \mathrm{VCO}_{2}{ }^{-1}$. The latter method has received the most support recently and has been found to be the best and most reliable estimate of LT (Caizzo et al, 1982). The VT data points (like the LT) have been chosen subjectively which has raised questions about the validity of these measurements.

The LT and VT have been found to be altered by

Address for correspondence:

Dr. W. Larry Kenney

Laboratory for Human Performance Research

119 Noll Laboratory

The Pennsylvania State University

University Park

PA 16802

USA substrate availability and glycogen depletion. Both of these measures have been found (a) to correlate highly with endurance performance [e.g. Kumagai et al, 1982 ( $r=0.95$ ), Farrell et al, $1979(r=0.97)$, and Powers et al, 1983 $(r=0.94)]$, and (b) to increase with endurance training (Davis et al, 1979; Poole and Gaesser, 1985).

Only a few studies have examined the anaerobic threshold using steady state exercise. Whipp and Wasserman (1972) conducted constant load tests to evaluate oxygen uptake kinetics. They concluded that a delay in attaining steady state $\mathrm{VO}_{2}$ of greater than 3 minutes indicated that the subject was working outside the aerobic level. Conversely, if a delayed steady state was not observed, the subject was exercising at or below anaerobic threshold. The highest workload at which this steady state was not delayed was termed the $\mathrm{MSSVO}_{2}$. MSSLA was measured and defined by Heck et al (1985) as the maximum workload at which there is a balance between lactate production and elimination.

The purpose of this investigation was to compare $L T, V T$, MSSLA, and $\mathrm{MSSVO}_{2}$ (expressed both as $\mathrm{VO}_{2}$ and running velocity) and to determine the relationship of these variables to $5 \mathrm{~km}$ running performance.

\section{METHODS}

The subjects for this study were 11 male recreational runners. Their physical characteristics are shown in Table I. Each subject underwent an incremental treadmill test to exhaustion. The protocol consisted of a 4-minute warm-up

TABLE I

Physiological characteristics of the subjects

\begin{tabular}{lccccc}
\hline & $\begin{array}{c}\text { Age } \\
(\mathrm{yrs})\end{array}$ & $\begin{array}{c}\text { Height } \\
(\mathrm{cm})\end{array}$ & $\begin{array}{c}\text { Weight } \\
(\mathrm{kg})\end{array}$ & $\begin{array}{c}\text { Body Fat } \\
(\%)\end{array}$ & $\begin{array}{c}\mathrm{VO}_{2} \text { max } \\
\left(\mathrm{ml}^{\prime} \mathrm{kg}^{-1} \mathrm{~min}^{-1}\right)\end{array}$ \\
\hline Mean & 22.4 & 178.0 & 71.29 & 13.52 & 57.2 \\
SD & 3.6 & 7.7 & 6.27 & 5.17 & 4.6 \\
Range & $18-28$ & $164.6-189.0$ & $60.0-81.1$ & $2.8-19.8$ & $49.9-65.3$ \\
\hline
\end{tabular}


at $2.41 \mathrm{~m} . \mathrm{s}^{-1}, 0 \%$ grade, with speed increases of $0.27 \mathrm{~m} . \mathrm{s}^{-1}$ every 2 minutes thereafter. Expired gas was collected and analysed every 20 seconds by a Gould -9000 computerised pulmonary work station. The subjects breathed room air through a low-resistance, one-way valve; flow was measured by a rolling seal spirometer system. Analysers were calibrated prior to each test. This system calculated $\mathrm{VO}_{2}, \mathrm{VE}$, and $\mathrm{VCO}_{2}$ every 20 seconds. Heart rate was recorded every minute from an ECG (Hewlett Packard).

Arterialised capillary blood (Forster et al, 1972) was sampled by finger-prick every 2 minutes during each test and analysed enzymatically for lactate using a Lactate/640 Analyser. A wet, warm cloth was placed on the finger while the subject was running to maintain blood flow. Subjects required one to four blood samples per test and all sampling was done while the subject continued to run. Blood was collected in heparinised capillary tubes and immediately placed into a standardised diluting solution. Samples were analysed within 24 hours or frozen and analysed within 5 days. The LT was determined subjectively by three experienced evaluators as the point at which a systematic increase in lactate began. The VT, defined as an increase in $\mathrm{VE}^{\mathrm{VOO}}{ }_{2}^{-1}$ without a rise in $\mathrm{VE} . \mathrm{VCO}_{2}{ }^{-1}$ was determined subjectively by 4 evaluators. $\mathrm{VO}_{2} \max$ was determined as the highest $\mathrm{VO}_{2}$ attained during the test.

A series of 20-minute steady state tests were conducted for each subject. Gas collection and analysis, blood sampling and analysis, and heart rate recording were done as in the incremental tests. Testing at various velocities was continued until MSSLA and $\mathrm{MSSVO}_{2}$ were determined. MSSLA was determined as the highest workload at which lactate did not rise more than $0.2 \mathrm{mmol}^{-1} \mathrm{I}^{-1}$ between minute 10 and 20; $\mathrm{MSSVO}_{2}$ was determined as the highest workload at which a steady state in $\mathrm{VO}_{2}$ was reached by minute 3. A steady state was defined as the average $\mathrm{VO}_{2}$ over the last 10 minutes $\pm 0.21 . \mathrm{min}^{-1}$.

Each subject also ran a $\mathbf{5} \mathbf{~ k m}$ time trial on a track and performance times were recorded. A one-way analysis of variance was used to compare the four physiological variables, MSSLA, MSSVO $2, L T$, and VT. A multiple correlation analysis was run on all variables including the $5 \mathrm{~km}$ performance. A step-wise regression was performed to determine if combined physiological variables could predict $5 \mathrm{~km}$ performance. All methods were approved by the Committee for the Protection of Human Subjects at The Pennsylvania State University.

\section{RESULTS}

Results of all treadmill tests and of the $5 \mathrm{~km}$ run are presented in Table II. None of the test variables, i.e. LT, VT, MMSLA, and $\mathrm{MSSVO}_{2}$, differed significantly from any other, on analysis of variance. However, for any given subject, the four were often very different (e.g. subject 8). As the $5 \mathrm{~km}$ results illustrate, the subjects were well-trained and used to competitive running, with $5 \mathrm{~km}$ times in this trial ranging from 15.3 to 20.6 minutes. There was no relationship between $\mathrm{VO}_{2}$ max and $5 \mathrm{~km}$ performance $\left(r^{2}=0.02\right)$.

Reliability of the data collected in this study was high, as expected from previous studies (Hughes et al, 1982; Reinhard et al, 1979). A test-retest correlation analysis for 12 simultaneously-drawn blood lactate samples yielded a correlation coefficient of $r=0.99$. Fig. 1 shows test-retest results from one subject on separate days with respect to $\mathrm{VO}_{2}$ and lactate measurement.

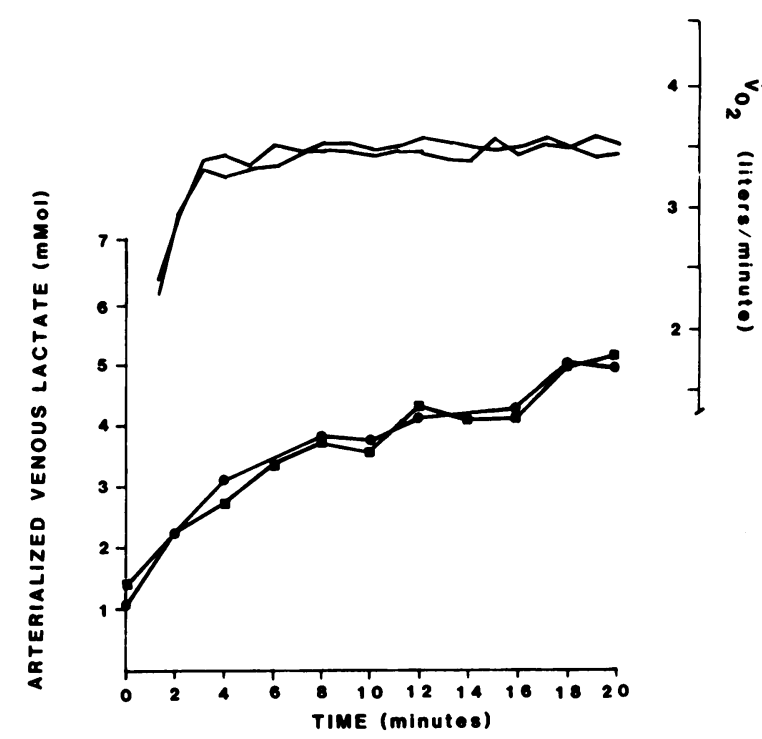

Fig. 1: Test-retest plot of arterialised venous lactate and $\mathrm{VO}_{2}$ values from one subject exercise at steady state intensity on separate days.

Representative data from one subject are plotted in Fig. 2 (LT and VT as determined from the incremental tests) and Fig. 3 (MSSLA and $\mathrm{MSSVO}_{2}$ as determined from the series of steady state runs). Inter-investigator agreement was good, as shown in Table III for the LT selections.

In general, the variables were better related when expressed as running velocity. Again, the two steady state variables were highly correlated $(r=0.90)$. When expressed as velocity, both VT $(r=0.60)$ and MSSLA $(r=0.90)$ were related to performance suggesting that high VT or MSSLA velocities are related to better performance times (higher average velocity) in a $5 \mathrm{~km}$ run.

\section{DISCUSSION}

The results of this study suggest that there exists a close relationship between the objectively determined variables MSSLA and $\mathrm{MSSVO}_{2}$. This may be explained by the

TABLE ॥

Results of treadmill running tests and $5 \mathrm{~km}$ run

\begin{tabular}{|c|c|c|c|c|c|c|c|c|c|c|c|c|c|}
\hline & & LT & & & VT & & & MSSLA & & & $\mathrm{MSSVO}_{2}$ & & $5 \mathrm{~km}$ run \\
\hline & $\mathrm{VO}_{2}^{*}$ & $\% \mathrm{VO}_{2} \max$ & ms. ${ }^{-1}$ & $\mathrm{VO}_{2}^{*}$ & $\% \mathrm{VO}_{2} \max$ & $\mathrm{m} \cdot \mathrm{s}^{-1}$ & $\mathrm{vO}_{2}^{*}$ & $\% \mathrm{VO}_{2} \max$ & $\mathrm{m} \cdot \mathrm{s}^{-1}$ & $\mathrm{VO}_{2}^{*}$ & $\% \mathrm{VO}_{2} \max$ & $\mathrm{m} \cdot \mathrm{s}^{-1}$ & $\mathbf{m} \cdot \mathbf{s}^{-1}$ \\
\hline Mean & 41.59 & 73.0 & 3.44 & 43.2 & 76.3 & 3.62 & 44.9 & 78.6 & 3.75 & 45.9 & 80.0 & 3.84 & 4.56 \\
\hline SD & 3.74 & 7.3 & 0.27 & 2.5 & 3.9 & 0.31 & 3.9 & 6.7 & 0.36 & 4.0 & 6.6 & 0.36 & 0.49 \\
\hline Range & $37.0-46.8$ & $59.8-84.9$ & $2.95-3.75$ & $39.5-48.2$ & $68.8-81.0$ & $3.22-3.84$ & $37.9-52.3$ & $67.4-90.3$ & $3.22-4.29$ & $37.9-49.2$ & $67.4-90.3$ & $3.22-4.29$ & $4.02-5.45$ \\
\hline
\end{tabular}

$* \mathrm{VO}_{2}$ values expressed in $\mathrm{ml} \mathrm{kg}^{-1} \mathrm{~min}^{-1}$ 

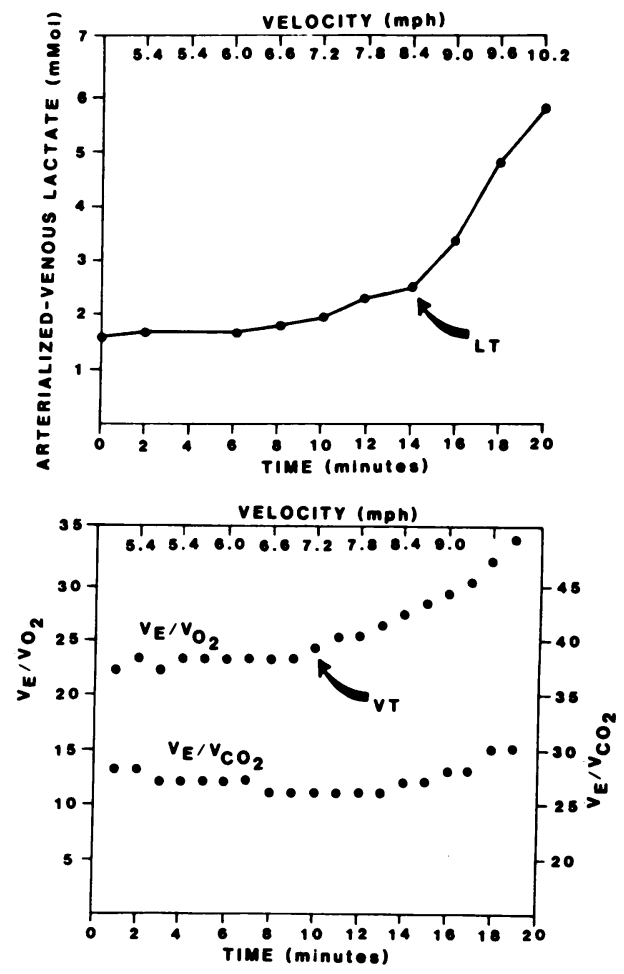

Fig. 2: Sample plots of arterialised venous lactate and respiratory parameters used to determine $L T$ and VT, respectively (see text for description of threshold determination).

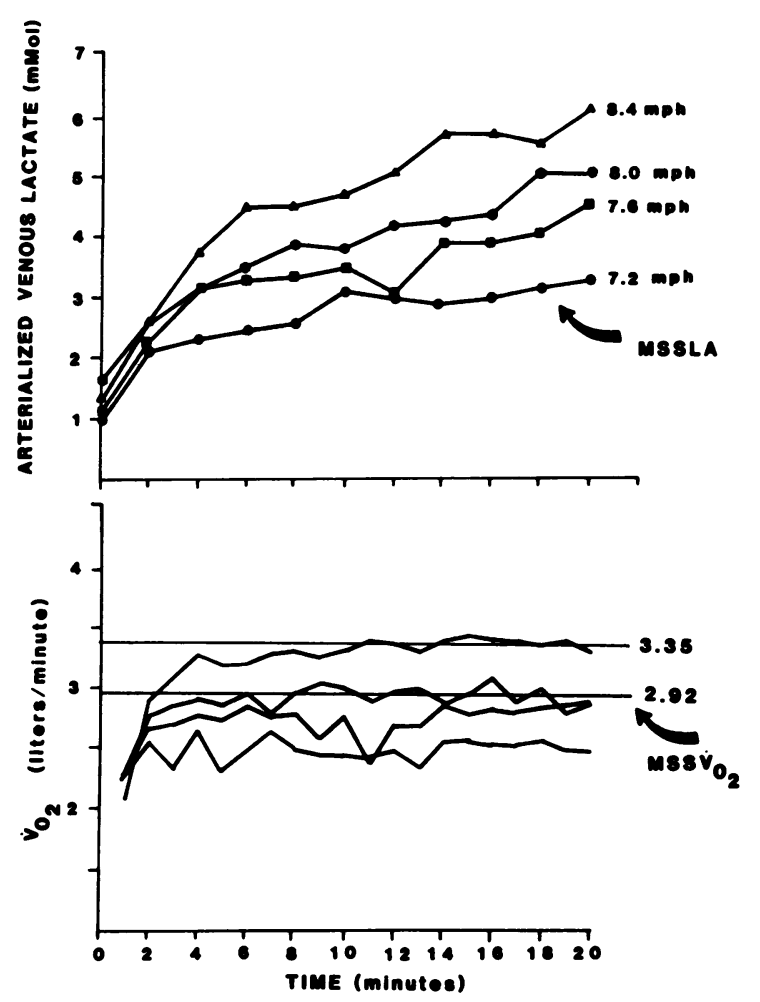

Fig. 3: Sample plots of data from a representative subject used to determine MSSLA and $\mathrm{MSSVO}_{2}$ (see text for description).

relationship of lactate production and elimination to $\mathrm{VO}_{2}$. When a subject is working at a low-to-moderate intensity, lactate diffuses into the blood and can be traced in the venous drainage from the muscle. As exercise proceeds, lactate is maintained at a fairly constant concentration in the blood (Åstrand and Rodahl, 1986, p. 320). At such low
TABLE III

Investigator data point selection for LT (speed in $\mathbf{m . s ^ { - 1 }}$ )

\begin{tabular}{rcccc}
\hline Subject & Investigator & Investigator & Investigator & $\begin{array}{c}\text { Value } \\
\text { Used }\end{array}$ \\
\hline 1 & 1 & 2 & 3 & \\
2 & 3.75 & 3.75 & 3.75 & 3.75 \\
3 & 3.49 & 3.53 & 3.22 & 3.49 \\
4 & 3.40 & 3.40 & 3.22 & 3.40 \\
5 & 2.95 & 2.91 & 2.95 & 2.95 \\
6 & 3.75 & 3.53 & 3.75 & 3.75 \\
7 & 3.75 & 3.84 & 3.75 & 3.75 \\
8 & 3.22 & 3.31 & 3.22 & 3.22 \\
9 & 3.49 & 3.49 & 3.49 & 3.49 \\
10 & 3.22 & 3.26 & 3.22 & 3.22 \\
11 & 3.49 & 3.22 & 3.49 & 3.49 \\
& 3.49 & 3.22 & 3.49 & 3.49 \\
\hline
\end{tabular}

intensities, the subject is also able to attain a steady state in $\mathrm{VO}_{2}$ within 3 minutes (Åstrand and Rodahl, 1986, p. 299; Whipp and Wasserman, 1972). During more intense work, lactate is produced faster than it can be eliminated and $\mathrm{VO}_{2}$ steady state takes longer than 3 minutes to reach. In the early minutes of constant-load exercise, energy needs may be met to a greater extent by anaerobic processes. Lactate is produced and $\mathrm{VO}_{2}$ is low. As the subject adjusts to the workload, aerobic processes contribute a greater proportion of the energy as $\mathrm{VO}_{2}$ reaches a steady state and lactate concentration plateaus (Åstrand and Rodahl, 1986). The simultaneous occurrence of these two processes may account for the high relationship between MSSLA and $\mathrm{MSSVO}_{2}$.

VT has been said to occur due to the increase of VE (the result of an increased buffering of lactate by the bicarbonate system, increasing $\mathrm{VCO}_{2}$ ) (Wasserman et al, 1973). However, buffering may not occur significantly until lactate in the blood reaches a critical concentration which may not correspond to the LT. A second potential reason for the lack of agreement between LT and VT is the inconsistency in incremental testing protocols across studies (Hughes et al, 1982). This is most apparent when measuring LT. It must be recognised that it takes some time (possibly different for each person) for lactate to reach a steady state, therefore some subjects may require a few more minutes at each workload for lactate to be eliminated. An over- or underestimated LT could occur if, indeed, LT is dependent on the type of protocol used, i.e. length of each increment.

Much higher relationships were found when variables were expressed as velocity rather than $\mathrm{VO}_{2}$. This may be explained by the differences in running economy between subjects where running economy is defined as the $\mathrm{VO}_{2}$ cost of running at a fixed intensity (Cavanagh and Kram, 1982). $\mathrm{VO}_{2}$ values were collected for 8 subjects at speeds of $3.75 \mathrm{~m} . \mathrm{s}^{-1}$ and $3.92 \mathrm{~m} . \mathrm{s}^{-1}$ for the steady state tests. At $3.75 \mathrm{~m} . \mathrm{s}^{-1}$, there were large differences in running economy with $\mathrm{VO}_{2}$ ranging from 34.9 to $48.4 \mathrm{ml}^{.} \mathrm{kg}^{-1} \mathrm{~min}^{-1}$. The faster $5 \mathrm{~km}$ runners do not necessarily have better economy. For instance, subjects 5 and 6 had almost identical performance times, yet economy differed by

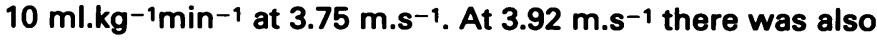
a great deal of variability in ${\nabla \mathrm{O}_{2}}_{2}$ however the most

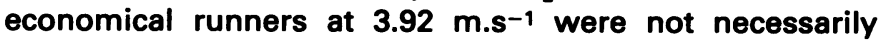

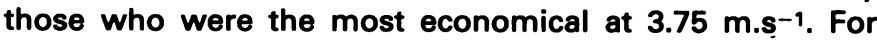
example, subject 4, the most economical runner at $3.75 \mathrm{m.s}^{-1}$, was only the 4 th most economical at 3.92 m.s. ${ }^{-1}$. 
Running economy was unrelated to $\mathrm{VO}_{2}$ max in this sample.

TABLE IV

Correlation matrix for physiological variables and performance $\left(\mathrm{VO}_{2}\right)$

\begin{tabular}{|c|c|c|c|c|}
\hline & LT & $V T$ & MSSLA & $\mathrm{MSSVO}_{2}$ \\
\hline \multicolumn{5}{|l|}{ LT } \\
\hline $\mathrm{VT}$ & 0.34 & & & \\
\hline MSSLA & -0.47 & 0.37 & & \\
\hline $\mathrm{MSSVO}_{2}$ & -0.31 & 0.44 & $0.74^{* *}$ & \\
\hline $5 \mathrm{~km}$ & -0.56 & 0.56 & 0.54 & 0.57 \\
\hline
\end{tabular}

* *Significant at $p<0.01$

The best predictor of $5 \mathrm{~km}$ performance as the MSSLA velocity $(r=0.87)$. This variable has also been measured by Heck et al (1985), and was found to be similar to the velocity corresponding to a blood lactate concentration of $4 \mathrm{mmol} . \mathrm{I}^{-1}$ during incremental tests. Our MSSLA lactate values were somewhat lower than $4 \mathrm{mmol}^{-\mathrm{I}^{-1}}\left(2.8 \pm 1.0 \mathrm{mmol} . \mathrm{I}^{-1}\right)$ and were not significantly different from the $L T$ values $(2.3 \pm 0.5$ mmol.I-1). However, 8 of the 11 runners had higher lactate levels at MSSLA than at LT. Heck et al (1985), however, found a poor correlation between treadmill performance and MSSLA. The present study used a much more conservative criterion for a steady state in lactate (0.2 mmol. $\left.\mathrm{I}^{-1}\right)$ than Heck et al $\left(1.0 \mathrm{mmol} . \mathrm{I}^{-1}\right)$. The present study found that faster runners tend to have higher MSSLA values, suggesting that MSSLA may be an important factor in describing cardiorespiratory endurance. Above MSSLA, lactate continues to be produced faster than it is eliminated. This build-up of lactate may cause the athlete to discontinue effort at this intensity due to (a) the inability to eliminate lactate, (b) the excessive production of lactate, and/or (c) the inability to tolerate lactate. The slighly higher lactate levels at MSSLA than at LT are consistent with higher (close to practical?) running speeds. As performance is presumably limited by lactate accumulation over a distance of $5 \mathrm{~km}$, the MSSLA might be expected to be a better predictor of performance than LT. Such was the case here.

TABLE V

Correlation matrix for physiological variables and performance (speed)

\begin{tabular}{lrlll}
\hline & LT & VT & MSSLA & MSSVO $_{2}$ \\
\hline LT & & & & \\
VT & 0.32 & & & \\
MSSLA & -0.01 & $0.67^{*}$ & & \\
MSSVO, & 0.16 & $0.70^{*}$ & $0.90^{* *}$ & 0.57 \\
5 km & 0.06 & $0.60^{*}$ & $0.87^{* *}$ & \\
\hline
\end{tabular}

"Significant at $\mathrm{p}<0.05 ;{ }^{* *} \mathrm{p}<0.01$

All of the runners in this study ran a $5 \mathrm{~km}$ time trial considerably faster than any of the velocities corresponding to the physiological variables LT, VT, MSSLA, or MSSVO ${ }_{2}$. The MSSLA may correspond more closely to a pace which can be sustained for prolonged periods, e.g. a marathon. For $5 \mathrm{~km}$, the subjects were presumably able to tolerate a higher blood lactate concentration than MSSLA. Alternately, it is possible that subjects may have been able to aid the lactate elimination process by slowing down and speeding up throughout the run.

The MSSLA represents the highest exercise intensity at which a continued increase in lactic acidosis does not occur. This may not correspond to the anaerobic threshold defined as the intensity just below the onset of metabolic acidosis (Stegmann et al, 1981). However, MSSLA may be a valuable measure and was found in this study to be objective and reliable.

In summary, mean values for the physiological variables LT, VT MSSLA, and $\mathrm{MSSVO}_{2}$ were found not to be significantly different from one another in a group of 11 trained runners. On the other hand, these values were often quite different within subjects. The best inter-relationship was found between MSSLA and $\mathrm{MSSVO}_{2}$. The best predictor of $5 \mathrm{~km}$ performance was MSSLA expressed in velocity. The MSSLA may be an important measure of cardiorespiratory endurance and warrants further study.

\section{References}

Astrand, P.-O. and Rodahl, K., 1986. Textbook of Work Physiology, 3rd ed. McGraw-Hill Book Co., New York.

Caizzo, V. J., Davis, J. A., Ellis, J. F., Azus, J. L., Vardagriff, R., Preitto, C. A. and McMaster, W. C., 1982 “A comparison of gas exchange indices to detect the anaerobic threshold". J.Appl.Physiol. 53 (5): 1184-1189.

Cavanagh, P. R. and Kram, R., 1982 "The efficiency of human movement - a statement of the problem". Med.Sci.Sports Exerc. 17 (3): 304-308.

Davis, J. A., Vodak, P., Wilmore, J. H., Vodak, J. and Kurtz, P., 1976 "Anaerobic threshold and maximal aerobic power for three modes of exercise". J.Appl.Physiol. 41 (4): 544-550.

Davis, J. J., Frank, M. H., Whipp, B. J. and Wasserman, K., 1979 "Anaerobic threshold alterations caused by endurance training in middle-aged men". J.Appl.Physiol. 46: 1039-1046.

Farrell, P. A., Wilmore, J. H., Coyle, F., Billings, J. E. and Costill, D. L., 1979 "Plasma lactate accumulation and distance running performance". Med.Sci.Sports Exerc. II: 338-344.

Forster, H. V., Dempsey, J. A., Thomson, J., Vidruk, E. and de Pico, G. A., 1972 "Estimation of arterial $\mathrm{PO}_{2}, \mathrm{pH}$, and lactate from arterialized venous blood". J.Appl.Physiol. 32: 134-137.

Green, H. J., Hughson, R. L., Orr, W. and Ranney, D. A., 1983 "Anaerobic threshold, blood lactate, and muscle metabolites in progressive exercise". J.Appl.Physiol. 54: 1032-1038.

Heck, H., Mader, A., Hess, G., Mucke, S., Muller, R. and Hollomann, W., 1985 "Justification of the $4.0 \mathrm{mmol}$. lactate threshold". Int.J.Sports Med. 6: 117-130.

Hughes, E. F., Turner, S. C. and Brooks, G. A., 1982 "Effects of glycogen depletion and pedalling speed on 'anaerobic threshold" ". J.Appl.Physiol. 52: 1598-1607.

IVy, J. L., Withers, R. T., Van Handel, P. J., Elger, D. H. and Costill, D. L., 1980 "Muscle respiratory capacity and fiber types as determinants of the lactate threshold". J.Appl.Physiol. 48: 523-527.

IVy, J. L., Costill, D. L., Van Handel, P. J., Essig, D. H. and Lower, R. W., 1981 "Alteration in the lactate threshold with changes in substrate availability". Int.J.Sports Med. 2 (3): 139-149.

Kumagai, S., Tanaka, S., Matsuura, Y., Matsuzaka, A. and Hirakoba, K, 1982 "Relationships of the anaerobic threshold with the $5 \mathrm{~km}, 10 \mathrm{~km}$, and 10 mile races". Eur.J.Appl.Physiol. 49: 13-23.

McLellan, T. M., 1985 "Ventilatory and plasma lactate response with different exercise protocols: A comparison of methods". Int.J.Sports Med. 6 (1): 30-35.

Poole, D. C. and Gaesser, G. A., 1985 "Response of ventilatory and lactate thresholds to continuous and interval training". J.Appl.Physiol. 58: 1115-1121.

Powers, S. K., Dodd, S., Deadson, R., Byrd, R. and McKnight, T., 1983 "Ventilatory threshold, running economy, and distance running performance of trained athletes". Res.Quart.Exerc.Sport 54: 179-182.

Reinhard, U., Muller, P. H. and Schmulling, R. H., 1979 "Determination of anaerobic threshold by the ventilation equivalent in normal individuals". Respiration 38: 36-42.

Stegmann, H., Kinderman, W. and Schnabel, A., 1981 "Lactate kinetics and the individual anaerobic threshold". Int.J.Sports Med. 2: 160-165.

Wasserman, K., Whipp, B. J., Koyal, S. N. and Beaver, W. L., 1973 "Anaerobic threshold and respiratory gas exchange during exercise". J.Appl.Physiol. 35: 236-243.

Whipp, B. J. and Wasserman, K., 1972 "Oxygen uptake kinetics for various intensities of constant load work". J.Appl.Physiol. 33: 351-356. 